\title{
Electron-phonon contribution to the phonon and excited electron (hole) linewidths in bulk Pd
}

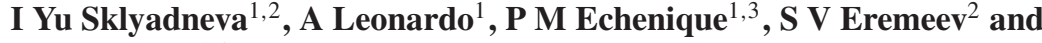 \\ E V Chulkov ${ }^{1,3}$ \\ ${ }^{1}$ Donostia International Physics Center (DIPC), 20018 San Sebastián/Donostia, Basque Country, \\ Spain \\ 2 Institute of Strength Physics and Materials Science, pr. Academicheski 2/1, 634021, Tomsk, \\ Russia \\ ${ }^{3}$ Departmento de Física de Materiales and Centro Mixto CSIC-UPV/EHU, Facultad de Ciencias \\ Químicas, UPV/EHU, Apartado 1072, 20080 San Sebastián/Donostia, Basque Country, Spain \\ E-mail: swxskski@sc.ehu.es
}

Received 30 March 2006

Published 7 August 2006

Online at stacks.iop.org/JPhysCM/18/7923

\begin{abstract}
We present an $a b$ initio study of the electron-phonon (e-ph) coupling and its contribution to the phonon linewidths and to the lifetime broadening of excited electron and hole states in bulk Pd. The calculations, based on density-functional theory, were carried out using a linear-response approach in the plane-wave pseudopotential representation. The obtained results for the Eliashberg spectral function $\alpha^{2} F(\omega)$, e-ph coupling constant $\lambda$, and the contribution to the lifetime broadening, $\Gamma_{\mathrm{e}-\mathrm{ph}}$, show strong dependence on both the energy and momentum of an electron (hole) state. The calculation of phonon linewidths gives, in agreement with experimental observations, an anomalously large broadening for the transverse phonon mode $T_{1}$ in the $\Sigma$ direction. In addition, this mode is found to contribute most strongly to the electron-phonon scattering processes on the Fermi surface.
\end{abstract}

\section{Introduction}

Electron-phonon interaction (e-ph) in metals is a subject of intensive theoretical and experimental investigation. The interest in this problem arises from a variety of physical phenomena which appear to be determined by phonons and their interactions with electrons. In particular, the electron-phonon coupling causes the enhancement of the effective electron mass (renormalization of the electronic specific heat), contributes to transport properties such as electrical and thermal resistivities, and plays an important role in superconductivity [1-3]. It also contributes to a finite phonon lifetime [2] as well as to lifetimes of excited electrons and holes $[4,5]$.

A central quantity of the electron-phonon interaction is the Eliashberg spectral function $\alpha^{2} F(\omega)$, which measures the contribution of phonons with frequency $\omega$ to the scattering of 
electrons [6]. The strength of the e-ph interaction is described by a dimensionless coupling parameter $\lambda$ which is proportional to the inverse frequency moment of $\alpha^{2} F(\omega)$. Both $\alpha^{2} F(\omega)$ and $\lambda$ at the Fermi energy $\left(\epsilon_{\mathrm{F}}\right)$ can be measured for superconducting materials by tunnelling spectroscopy $[2,7]$. When the superconductivity is absent, $\lambda$ can be extracted from specific heat measurements [2] or from the measured slope of the real part of the self-energy at $\epsilon_{\mathrm{F}}$ using photoemission spectroscopy (PES) [8-11]. For hole states located relatively far from the Fermi level, $\lambda$ can be deduced from PES measurements of the temperature dependence of a hole linewidth $[12,13]$. However, PES, with its unprecedented accuracy of measuring $\lambda$ in surface and quantum-well states [12-20], is not well suited for bulk materials because of the known final states effect [21]. Very detailed information concerning electron-phonon interactions can also be obtained from measurements of phonon linewidths. As is well known, the phonons and their characteristics in metals are strongly influenced by the e-ph coupling [22]. In particular, it was shown that the e-ph mass enhancement parameter $\left(\lambda\right.$ at $\left.\epsilon_{\mathrm{F}}\right)$ is simply a weighted average of phonon linewidths [23, 24].

In the case of $\mathrm{Pd}$, which is neither ferromagnetic nor superconducting in spite of its high magnetic susceptibility [25] and electronic density of states (DOS) at the Fermi level (as well as the large electronic mass enhancement) [26-28], the estimation of the e-ph coupling parameter is complicated by parallel processes of spin fluctuations [29, 30]. The observation of phonon linewidths is also difficult and only a few experimental studies have been reported [31, 32]. One of them, an inelastic-neutron scattering study, was made at low temperatures for selected longitudinal phonons in two symmetry directions, $\Delta$ and $\Lambda$ [32]. The data for transverse mode $T_{1}$ in the $\Sigma$ direction were obtained by correction of the neutron groups for instrumental resolution [31]. The measured values of linewidths showed several distinctive features. One of them is a sharp maximum along the $\Delta$ symmetry direction for the longitudinal mode. The other feature is an anomalous broadening for the $T_{1}$ phonon branch at all temperatures of the experiment. The only theoretical evaluation of the e-ph contribution to phonon linewidths in bulk Pd [33] was carried out with electron wave functions and energies computed by the Korringa-Kohn-Rostoker (KKR) method and the rigid-muffin-tin approximation for the eph matrix elements. Phonon frequencies and polarization vectors were taken from Born-von Kármán force constants fitted to the experimental data [34]. As for the e-ph contribution to the lifetime broadening of excited electrons and holes at different energies and momenta, no data is available. In this situation, valuable information can be obtained from $a b$ initio self-consistent calculations.

Here we present the results of an ab initio linear-response study of the phonon linewidths for phonon wavevectors along high symmetry directions and compare them with available experimental data. We have calculated the e-ph spectral function and coupling parameter $\lambda$ for electron and hole states in the energy interval from $-3 \mathrm{eV}$ to $+3 \mathrm{eV}$ (with respect to $\epsilon_{\mathrm{F}}$ ) and for different momenta. We have also evaluated self-consistently the phonon-induced contribution to the lifetime broadening (linewidth) of these states in bulk Pd and its dependence on energy and momentum, as well as on temperature.

The paper is organized as follows. In section 2 a short outline of the calculation method is given. In section 3 we present and discuss in detail the calculation results. Finally, the conclusions are drawn in section 4 .

\section{Theory}

The linewidth $\gamma_{\boldsymbol{q}, \nu}$ of the phonon mode $(\boldsymbol{q}, v)$ arising from e-ph interactions may be written as [24]:

$$
\gamma_{\boldsymbol{q}, v}=2 \pi \omega_{\boldsymbol{q}, v} \sum_{\boldsymbol{k}, i, f}\left|g\left(\boldsymbol{k}_{i}, \boldsymbol{k}_{f}, \boldsymbol{q}, \nu\right)\right|^{2} \delta\left(\epsilon_{\boldsymbol{k}_{i}}-\epsilon_{\mathrm{F}}\right) \delta\left(\epsilon_{\boldsymbol{k}_{f}}-\epsilon_{\mathrm{F}}\right) .
$$


Here, $\omega_{\boldsymbol{q}, v}$ is the phonon frequency and $g\left(\boldsymbol{k}_{i}, \boldsymbol{k}_{f}, \boldsymbol{q}, v\right)$ is the electron-phonon matrix element which refers to the scattering of an electron from the initial state $\boldsymbol{k}_{i}$ with the energy $\epsilon_{\boldsymbol{k}_{i}}$ to the final state $\boldsymbol{k}_{f}$ with the energy $\epsilon_{\boldsymbol{k}_{f}}$ by the phonon $(\boldsymbol{q}, v)$. The sum is carried out over all initial and final electron states on the Fermi surface. In equation (1) we apply the usual assumption that phonon energies are much smaller than the electronic energy scale, i.e. $\delta\left(\epsilon_{k_{i}}-\epsilon_{\mathrm{F}} \pm \omega_{q, v}\right) \approx \delta\left(\epsilon_{k_{i}}-\epsilon_{\mathrm{F}}\right)$ (the so-called quasi-elastic approximation). The linewidth $\gamma_{q, v}$ is related to the e-ph coupling parameter at the Fermi energy, $\lambda\left(\epsilon_{\mathrm{F}}\right)$, which determines the electronic mass enhancement [24]:

$$
\lambda\left(\epsilon_{\mathrm{F}}\right)=\sum_{\boldsymbol{q}, v} \frac{1}{\pi N\left(\epsilon_{\mathrm{F}}\right)} \frac{\gamma_{\boldsymbol{q}, v}}{\omega_{\boldsymbol{q}, v}^{2}}=\sum_{\boldsymbol{q}, v} \lambda_{\boldsymbol{q}, v}\left(\epsilon_{\mathrm{F}}\right),
$$

where $N\left(\epsilon_{\mathrm{F}}\right)$ is the electron density of states per atom and spin at the Fermi level.

For energies $\epsilon_{k_{i}} \neq \epsilon_{\mathrm{F}}$, the $\boldsymbol{k}$-dependent strength of the e-ph coupling, $\lambda\left(\epsilon_{\boldsymbol{k}_{i}}\right)$, is defined as the first inverse frequency moment of the spectral function $\alpha^{2} F\left(\epsilon_{k_{i}} ; \omega\right)$ [2]:

$$
\lambda\left(\epsilon_{\boldsymbol{k}_{i}}\right)=2 \int \frac{\alpha^{2} F\left(\epsilon_{\boldsymbol{k}_{i}} ; \omega\right)}{\omega} \mathrm{d} \omega .
$$

The momentum-dependent e-ph spectral function, $\alpha^{2} F\left(\epsilon_{k_{i}} ; \omega\right)$, gives the e-ph coupling between a given electron (hole) state $\boldsymbol{k}_{i}$ and all other final states $\boldsymbol{k}_{f}$ which differ in energy from the initial one by $\hbar \omega[2]$ :

$$
\alpha^{2} F\left(\epsilon_{\boldsymbol{k}_{i}} ; \omega\right)=\sum_{\boldsymbol{q}, v, f} \delta\left(\omega-\omega_{\boldsymbol{q}, v}\right)\left|g\left(\boldsymbol{k}_{i}, \boldsymbol{k}_{f}, \boldsymbol{q}, \nu\right)\right|^{2} \delta\left(\epsilon_{\boldsymbol{k}_{f}}-\epsilon_{k_{i}}\right) .
$$

The sum in equation (4) is carried out over all possible final electron states $\boldsymbol{k}_{f}$ and all possible phonon modes $(\boldsymbol{q}, v)$. As in equation (1), the quasi-elastic approximation is used. The definition (3) of $\lambda\left(\epsilon_{\boldsymbol{k}_{i}}\right)$, integrated at $\epsilon_{\mathrm{F}}$ over electron momenta $\boldsymbol{k}_{i}$, coincides with $\lambda\left(\epsilon_{\mathrm{F}}\right)$ of equation (2).

Finally, the phonon-induced linewidth $\Gamma_{\mathrm{e}-\mathrm{ph}}\left(\epsilon_{k_{i}}\right)$ of an excited electron (hole) state with momentum $\boldsymbol{k}_{i}$ and energy $\epsilon_{\boldsymbol{k}_{i}}$ can be obtained from the imaginary part of the electron-phonon self-energy [2]:

$$
\begin{aligned}
\Gamma_{\mathrm{e}-\mathrm{ph}}\left(\epsilon_{k_{i}}\right)= & 2 \operatorname{Im} \Sigma_{i}^{0}\left(\epsilon_{\boldsymbol{k}_{i}} ; T\right) \\
& =2 \pi \int_{0}^{\omega_{\mathrm{m}}} \alpha^{2} F\left(\epsilon_{k_{i}} ; \omega\right)\left[1-f\left(\epsilon_{k_{i}}-\omega\right)+f\left(\epsilon_{k_{i}}+\omega\right)+2 n(\omega)\right] \mathrm{d} \omega .
\end{aligned}
$$

Here, $f(\omega)$ and $n(\omega)$ are the Fermi and Bose distributions, respectively, and $\omega_{\mathrm{m}}$ is the maximum phonon frequency. At high temperatures, when $k_{\mathrm{B}} T$ is higher than the maximum phonon energy, one can rewrite equation (5) as [2]:

$$
\Gamma_{\mathrm{e}-\mathrm{ph}}\left(\epsilon_{k_{i}}\right)=2 \pi \lambda\left(\epsilon_{k_{i}}\right) \mathrm{k}_{\mathrm{B}} T .
$$

All calculations presented here were performed using the ab initio pseudopotential local density approximation formalism with a plane-wave (PW) basis. Phonon frequencies, polarization vectors and electron-phonon interactions were calculated within the densityfunctional perturbation theory [35] and the PWSCF code [36]. The electron-ion interaction in $\mathrm{Pd}$ atoms is described by a nonlocal norm-conserving pseudopotential [37] based on semi-relativistic all-electron calculations. The exchange and correlation energy functional is approximated by the form of [38]. In order to enhance transferability, the pseudopotential has been generated so as to reproduce accurately several atomic configurations, including two excited states $\left(\mathrm{d}^{9} \mathrm{~s}^{1}\right.$ and $\left.\mathrm{d}^{8} \mathrm{~s}^{2}\right)$, because of the promotion of electrons from $\mathrm{d}$ to $\mathrm{s}$ electronic levels that occur in the solid. The size of the basis set was restricted by the kinetic energy cutoff of 

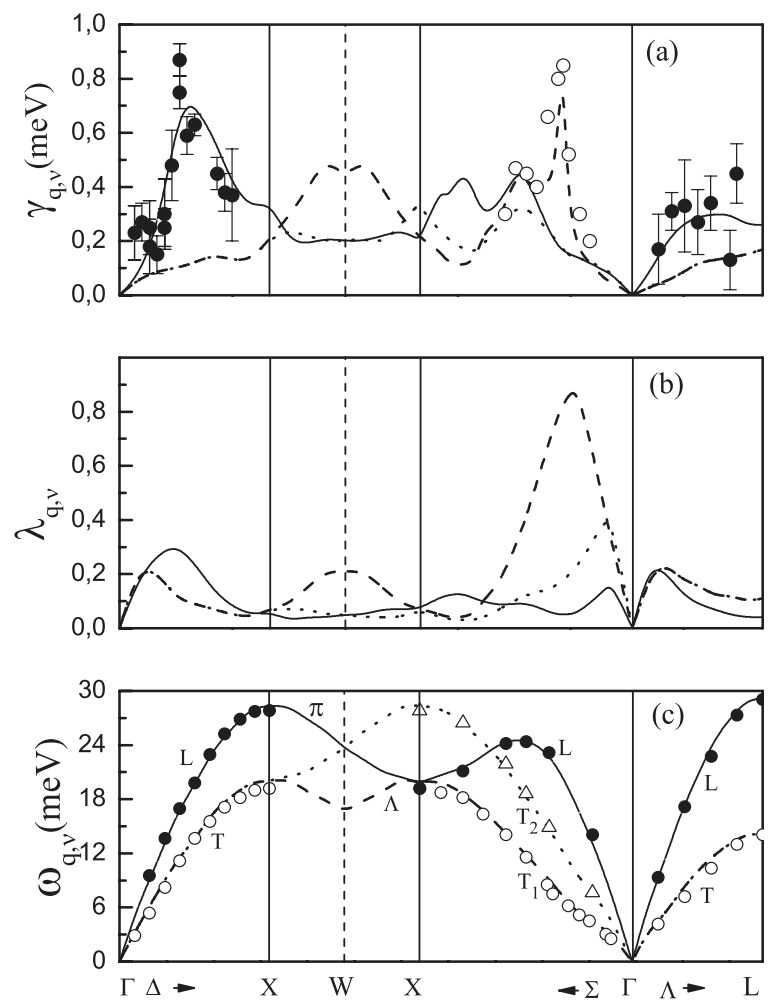

Figure 1. (a) Phonon linewidths $\gamma_{\boldsymbol{q}, v}$, (b) mass enhancement parameters $\lambda_{\boldsymbol{q}, v}$, and (c) phonon frequencies $\omega_{q, v}$ calculated along high symmetry directions. Full lines correspond to longitudinal modes, while dashed and dotted lines represent transverse branches. The available experimental data are shown by solid circles for longitudinal modes $[32,34]$ and by open circles and triangles for transverse phonons $[34,31]$.

$50 \mathrm{Ryd}(\approx 600 \mathrm{PW} /$ atom). The Brillouin zone (BZ) integrations were performed using the $\boldsymbol{k}$ space sampling [39] with 413 special $k$ points in the irreducible wedge of the BZ (IBZ). To account for the presence of a Fermi surface, we use the first-order Hermite-Gaussian smearing technique with a width of 0.02 Ryd [40].

\section{Results and discussion}

\subsection{Phonon linewidths}

First, we present the results of our calculation for the phonon linewidths $\gamma_{q, v}$. The $k$-space integration in equation (1) involving two $\delta$-functions was performed with a mesh of 413 points in the IBZ and then checked with $900 \boldsymbol{k}$ points. The latter number of $\boldsymbol{k}$ points was also used in the evaluation of the Eliashberg spectral function at $\epsilon_{\mathrm{F}}$ by Savrasov [41]. In both cases we obtained practically the same results. The $\delta$-functions were replaced by the first-order HermiteGaussian functions with width in the range of 0.01-0.03 Ryd. Since the set of $\boldsymbol{k}$ points sampled is large enough, the results are rather insensitive to the smearing width value. In figure 1 we display the wavevector- and branch-dependent $\gamma_{q, v}$ and $\lambda_{q, v}$ together with the phonon mode frequencies. In $\mathrm{Pd}$, the phonon dispersion curves qualitatively resemble those of other facecentred cubic (fcc) transition metals except for anomalous shifts in the frequencies for a phonon branch $T_{1}$ ( $\Sigma$ direction). Rather similar effects have also been observed in Pt [42]. Our results 
are in good agreement with experimental data [34] except for a very slight overestimation of the theoretical phonon frequencies at the zone boundary. As for the phonon linewidths, only a few measurements have been reported [31,32]. One of them was performed at low temperatures for longitudinal modes in the $\Delta$ and $\Lambda$ symmetry directions (figure 1(a), full circles) [32]. As one can see, both the experimental and theoretical data show a sharp maximum in the $\Delta$ direction. The position of the peak as well as the magnitude of the calculated phonon linewidths resemble well the experimental results. The values of $\gamma_{q, v}$ in bulk Pd obtained with the rigid-muffintin approximation for the el-ph matrix elements [33] give this peak at smaller $q$ than in the experiment [32]. In spite of the large broadening, the contribution of these phonon modes to the e-ph interactions on the Fermi surface (figure 1(b)) is not so large, although $\lambda_{q, v}$ shows a maximum at nearly the same position. The widths of transverse modes in the $\Delta$ and $\Lambda$ symmetry directions are much smaller compared to those for the longitudinal phonons. The appreciable values of $\gamma_{q, v}$ for transverse branches appear only at the zone boundary and along the $\Sigma$ direction where they have the same order of magnitude or are even larger than the widths of longitudinal modes. The most interesting feature is a peak in $\gamma_{q, v}$ for the phonon branch $T_{1}$. This result is in agreement with the data obtained experimentally by correction of the neutron groups for instrumental resolution [31]. The anomalous broadening was particularly evident at $8 \mathrm{~K}$, where the maximum linewidth was found to be $\sim 0.85 \mathrm{meV}$. Since anharmonic effects at these temperatures are expected to be negligible, one can suggest that the anomalous widths reflect mainly the reduction in phonon lifetimes. At higher temperatures, the large broadening was found to remain. However, it becomes somewhat smeared out, though the widths at these temperatures contain large contributions from the anharmonic damping of phonons. In addition, the $T_{1}$ transverse modes are found to contribute most strongly to the e-ph scattering processes on the Fermi surface.

It would be desirable to separate the mechanisms which determine the $\boldsymbol{q}$ and mode $v$ dependence of $\gamma_{q, v}$. However, it is difficult to evolve the effects of different factors in the first-principles calculation. Some conclusions can be drawn from considering the different transitions contributing to the e-ph scattering processes on the Fermi surface [33]. Thus, the prominent peak in the $\Delta$ direction for the longitudinal phonon branch was attributed to the scattering across the arms or 'pipes' of the Pd Fermi surface. The width of these 'pipes' in the (001) plane determines the position of the peak. It was also shown that the appearance of this peak is largely a matrix element effect which cannot be simply explained [33]. On the other hand, the transitions which contribute to the linewidths of the transverse modes in the $\Delta$ direction connect the points on the Fermi surface that are related by a mirror plane (001) and have vanishing matrix elements. As a result, their linewidths are rather small. As for the $T_{1}$ phonons in the $\Sigma$ symmetry direction, the main contributions to their linewidth come from the transitions that lie across the Fermi surface arms in the (001) plane [33]. To consider the $\left(\boldsymbol{k}_{i}, \boldsymbol{k}_{f}\right)$-dependence of the e-ph interaction on the Fermi surface, we have calculated the individual contributions to the e-ph coupling, $\lambda_{\boldsymbol{q}, v}\left(\boldsymbol{k}_{i}, \boldsymbol{k}_{f}\right)$, for the $T_{1}$ transverse phonons. The analysis has shown that the e-ph coupling is very strong only for certain pairs of $\left(\boldsymbol{k}_{i}, \boldsymbol{k}_{f}\right)$ : between states on the Fermi surfaces which lie near the $\Sigma$ symmetry direction. It is also interesting to note that the maximum in $T_{1}$ phonon linewidths coincides closely with the frequency shifts in this phonon branch (figure 1(c)). These anomalous lattice vibrations in $\mathrm{Pd}$ have been studied in detail at several temperatures between 85 and $673 \mathrm{~K}$ by inelastic neutron scattering [31]. The study revealed a rather abrupt change in slope of the dispersion curve which was found to be strongly temperature dependent, becoming smeared out with increasing temperature. It was also suggested that these anomalies arise from the Kohn effect [31].

In figure 2 we plot the Eliashberg spectral function $\alpha^{2} F(\omega)$ at $\epsilon_{\mathrm{F}}$ calculated from the phonon linewidths as a sum over contributions from all possible phonon modes with a 


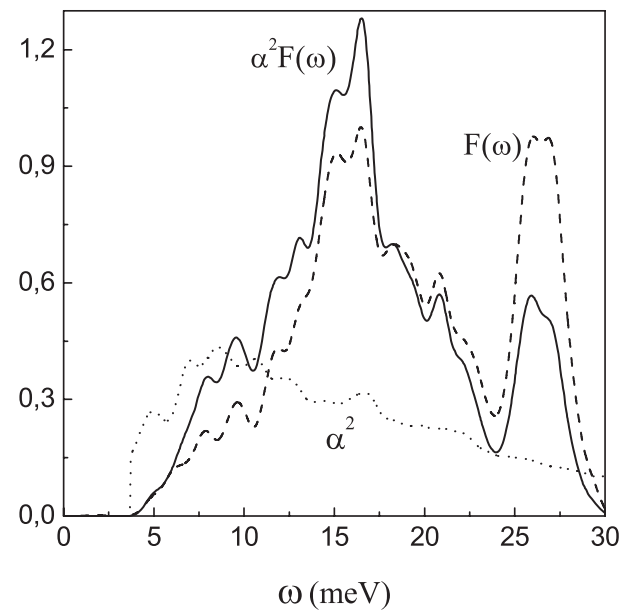

Figure 2. Phonon density of states $F(\omega)$ (dashed line) and electron-phonon spectral function $\alpha^{2} F(\omega)$ at $\epsilon_{\mathrm{F}}$ (solid line) normalized to enclose the same area. The frequency-dependent coupling function $\alpha^{2}$ (defined as the ratio $\left.\alpha^{2} F(\omega) / F(\omega)\right)$ is shown by dotted line.

frequency $\omega[24]$ :

$$
\alpha^{2} F(\omega)=\frac{1}{2 \pi N\left(\epsilon_{\mathrm{F}}\right)} \sum_{q, v} \frac{\gamma_{q, v}}{\omega_{q, v}} \delta\left(\omega-\omega_{q, v}\right) .
$$

Since the phonon linewidths $\gamma_{q, v}$ display rather significant variations throughout the BZ, the summation over the phonon wavevectors $\boldsymbol{q}$ and phonon branches $v$ in equation (7) was performed on rather dense meshes with 145 (and 256) points in the IBZ. The largest numerical error for $\alpha^{2} F(\omega)$ coming from the $q$ integration is found to be less than $1.6 \%$. Figure 2 also shows the calculated phonon density of states $F(\omega)$ (dashed line). In the case of Pd, the two curves are very similar in shape compared to those, for example, in bulk Be [43] where they are rather different, especially in the low-frequency region. This means that the coupling function $\alpha^{2}$ in Pd (also shown by a dotted line in the figure) is not strongly energy dependent. As one can see, the high-energy part of the spectrum is suppressed more by e-ph matrix elements than the lower-energy part. The same feature has been reported in [41], where the calculations were performed at the Fermi energy only. So, the contribution of low-energy phonons to the scattering processes of electrons on the Fermi surface is larger than that of high-frequency modes. This is a typical feature of noble and transition metals and is caused by the coupling of d electrons to transverse modes [44]. For a simple metal, like Be, with two atoms per unit cell, a different picture is observed [43]. In this case, the e-ph spectral function is dominated by high-frequency peaks which are determined by the scattering of optical phonons, while the low-energy peak in the phonon density of states is completely suppressed by the e-ph matrix elements. The electron-phonon mass enhancement parameter $\left(\lambda\right.$ at $\left.\epsilon_{\mathrm{F}}\right)$ for $\mathrm{Pd}$ was found to be $\lambda=0.40$. This value is very close to the value of $\lambda=0.41$ derived from KKR energies and wave functions with the rigid-muffin-tin approximation $[33,45]$ and is slightly higher $(14 \%)$ than $\lambda=0.35$ obtained from the first-principles linear-muffin-tin orbital calculation [41].

\subsection{Electron (hole) lifetime broadening}

Now we consider the contribution of the electron-phonon coupling to the linewidth (lifetime broadening) of excited electron and hole states and its variation with momentum and energy. 


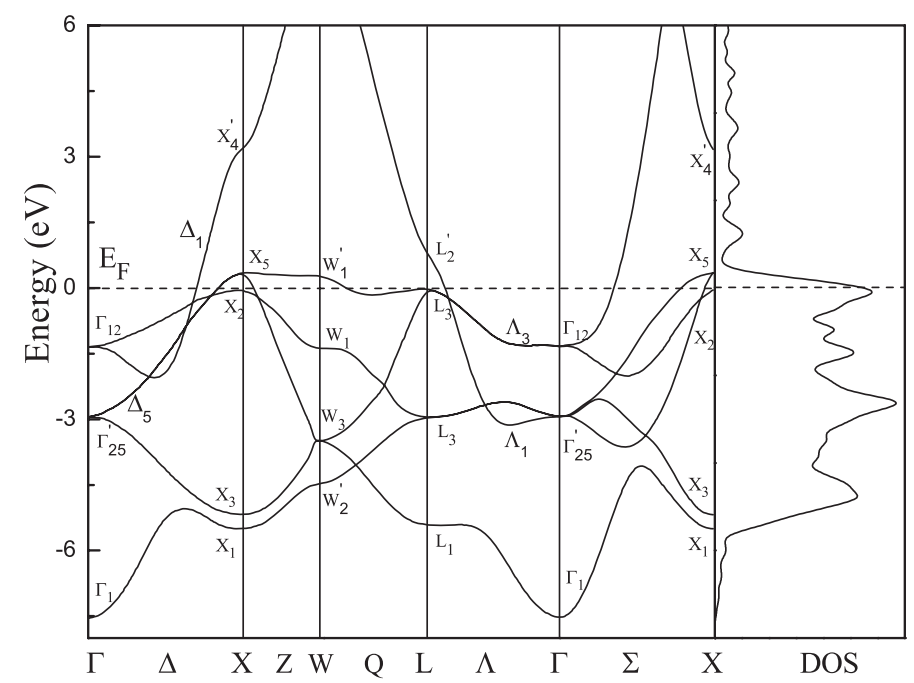

Figure 3. Electron energy bands for bulk Pd. The right panel shows the density of states.

In figure 3 we show the computed energy bands which are referred to the Fermi level. They agree well with both the available experimental data [46] and other first-principles calculations [47-49]. In particular, this conclusion was drawn from comparing the positions of the points arising from the crossing of bands with $\epsilon_{\mathrm{F}}$ and the electronic bands around the Fermi energy. Another characteristic feature is the density of states (DOS) at $\epsilon_{\mathrm{F}}$ per atom. We have obtained 32.16 states/Ryd, while the reported empirical value amounts to 31.8 states/Ryd [28]. The relativistic augmented-plane-wave calculations give $N\left(\epsilon_{\mathrm{F}}\right)=32.1$ states/Ryd [48] and 32.7 states/Ryd [48]. In other calculations, the value of the DOS was obtained in the range 31-34.14 states/Ryd [33, 41].

We have evaluated the phonon-induced linewidths, $\Gamma_{\mathrm{e}-\mathrm{ph}}\left(\epsilon_{\boldsymbol{k}_{i}}\right)$, and e-ph coupling, parameters $\lambda\left(\epsilon_{k_{i}}\right)$, as functions of energy for a number of states along the $\Delta_{1}, \Delta_{5}, \Lambda_{1}$, and $\Lambda_{3}$ energy bands. The top panels of figures 4 and 5 give the energy dependence of the lifetime broadening, $\Gamma_{\mathrm{e}-\mathrm{ph}}$, calculated at $T=0$. The middle panels of the figures show the variation of the electron-phonon coupling parameter. As follows from the figures, both functions display the same tendencies and vary very quickly, especially for the states in the $\Delta$ direction (figure 4). In the latter case, there are two pronounced maxima on moving from the $\Gamma$ point to the Fermi level (negative energies). One is reached around the centre of the symmetry direction and the other is situated near $\epsilon_{\mathrm{F}}$, at the maximum phonon energy. Above the Fermi level, the behaviour of the functions is quite different. For energies exceeding the maximum phonon energy, they go quickly down to zero and only near the $\mathrm{X}$ point a small increase is observed for the states in the $\Delta_{1}$ energy band (figures 4(b), (d)). One can note that, at some energies, $\Gamma_{\mathrm{e}-\mathrm{ph}}$ and $\lambda$ have two distinct values which correspond to two different momenta.

In figure 6 we show some of the calculated spectral functions $\alpha^{2} F\left(\epsilon_{k_{i}} ; \omega\right)$, in particular for the states with different energies along the $\Delta_{1}$ and $\Delta_{5}$ energy bands. As one can see, for $\Gamma_{25}^{\prime}$ and $\Gamma_{12}$ states (figures $6(\mathrm{a})$, (b), solid lines) most of the contribution to the electron-phonon coupling comes from the low-energy phonon modes. On moving to the Fermi level (dashed lines), the high-frequency peaks appear in the energy range of $25-28 \mathrm{meV}$. Their appearance in $\alpha^{2} F\left(\epsilon_{k_{i}} ; \omega\right)$ is determined by the coupling of the electron states at $\epsilon_{\mathrm{F}}$ with longitudinal phonons in the vicinity of the $X$ and $L$ symmetry points, as well as with high-frequency $T_{2}$ transverse 

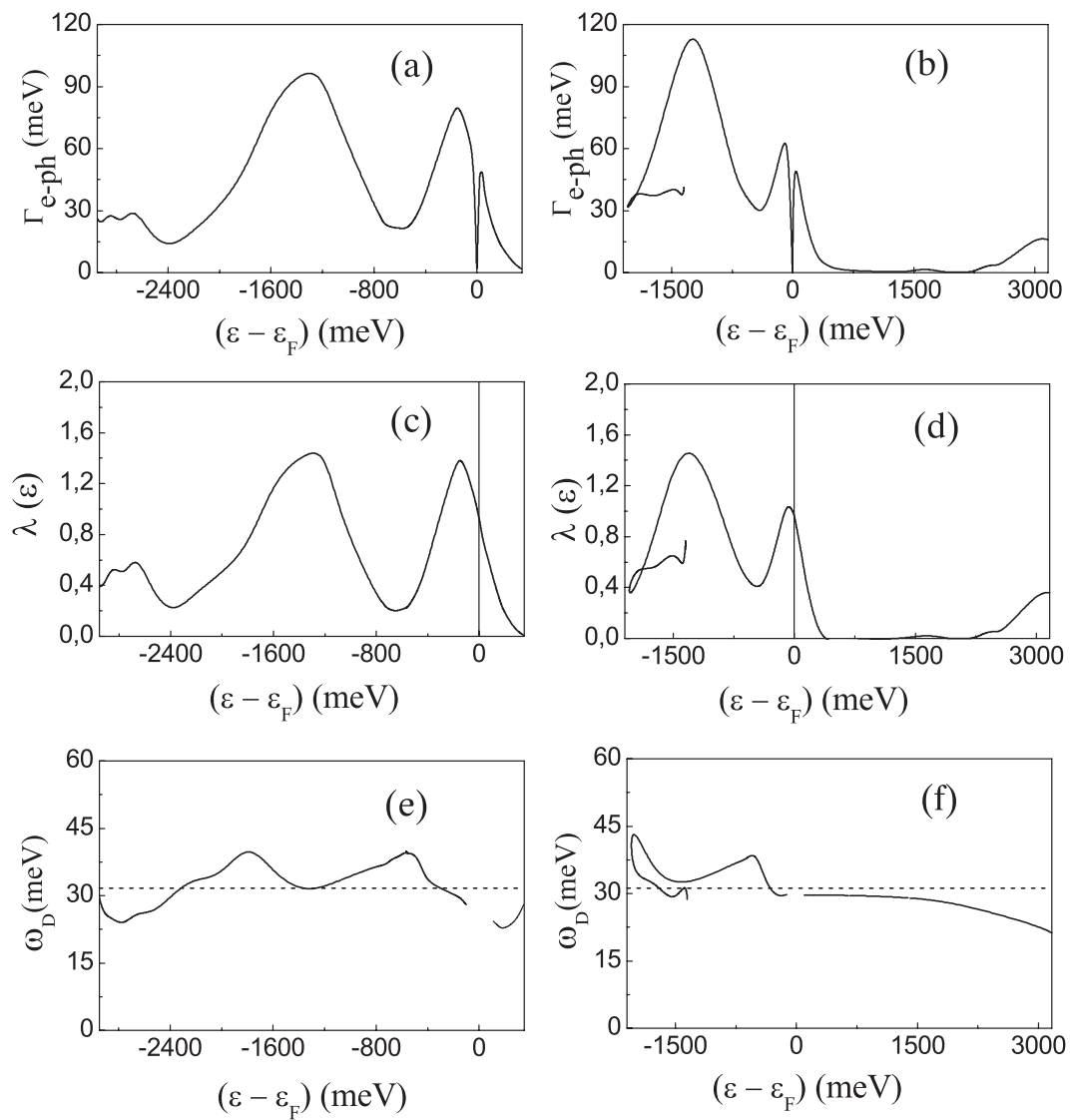

Figure 4. The $T=0$ phonon-induced lifetime broadening $\Gamma_{\mathrm{e}-\mathrm{ph}}$ and the electron-phonon coupling parameter $\lambda\left(\epsilon_{k_{i}}\right)$ as functions of energy for the $\Delta_{5}((\mathrm{a}),(\mathrm{c}))$ and $\Delta_{1}((\mathrm{~b}),(\mathrm{d}))$ energy bands, as well as the characteristic Debye frequency $\omega_{\mathrm{D}}((\mathrm{e}),(\mathrm{f}))$ calculated from equation $\Gamma_{\mathrm{e}-\mathrm{ph}}=2 \pi \lambda \hbar \omega_{\mathrm{D}} / 3$.

modes in the $Z$ and $\Sigma$ directions. Besides, in the case of the $\Delta_{5}$ energy band, the contributions from $T_{1}$ transverse modes (the $\Sigma$ symmetry direction) appear. As a result, the value of $\lambda\left(\epsilon_{\boldsymbol{k}_{i}}\right)$ increases on moving from the zone centre to the Fermi level and becomes nearly 2.5 times as large $\left(\lambda\left(\Gamma_{25}^{\prime}\right)=0.39\right.$ and $\left.\lambda\left(\epsilon_{\mathrm{F}}\right)=0.87\right)$. For the $\Delta_{1}$ energy band, the change in $\lambda\left(\epsilon_{k_{i}}\right)$ is not large and comes to 0.3 , because the e-ph coupling for the corresponding electron state at the zone centre, $\Gamma_{12}$, is not small, as it was in the previous case. It was found to be about two times as large compared to that for $\Gamma_{25}^{\prime}$. On the one hand, it is determined by the contributions coming from the scattering of the $T_{1}$ transverse modes (near the $\Sigma$ symmetry direction). On the other hand, the interaction with transverse modes propagating along the $\Lambda$ and $Z$ symmetry directions becomes much stronger than it was in the case of the $\Gamma_{25}^{\prime}$ electron state. We have also shown the spectral functions for two states at the $\mathrm{X}$ symmetry point: $\mathrm{X}_{5}$ and $\mathrm{X}_{4}^{\prime}$ (figure 6(c) solid and dashed lines, correspondingly). As one can see, for the $\Delta_{5}$ energy band the e-ph interaction becomes very weak near the $\mathrm{X}$ point where $\lambda\left(\mathrm{X}_{5}\right)=0.05$. In the case of $\Delta_{1}$, the e-ph coupling is not so small at the zone boundary, $\lambda\left(\mathrm{X}_{4}^{\prime}\right)=0.35$ and the calculated $\alpha^{2} F\left(\epsilon_{k_{i}} ; \omega\right)$ shows three prominent peaks. Two of them, at $5-6 \mathrm{meV}$ and at $\sim 7 \mathrm{meV}$, are mainly determined by the scattering of the $T_{1}$ transverse modes (near the $\Sigma$ direction). A small contribution to the first peak also comes from the scattering processes of longitudinal phonons propagating along the 

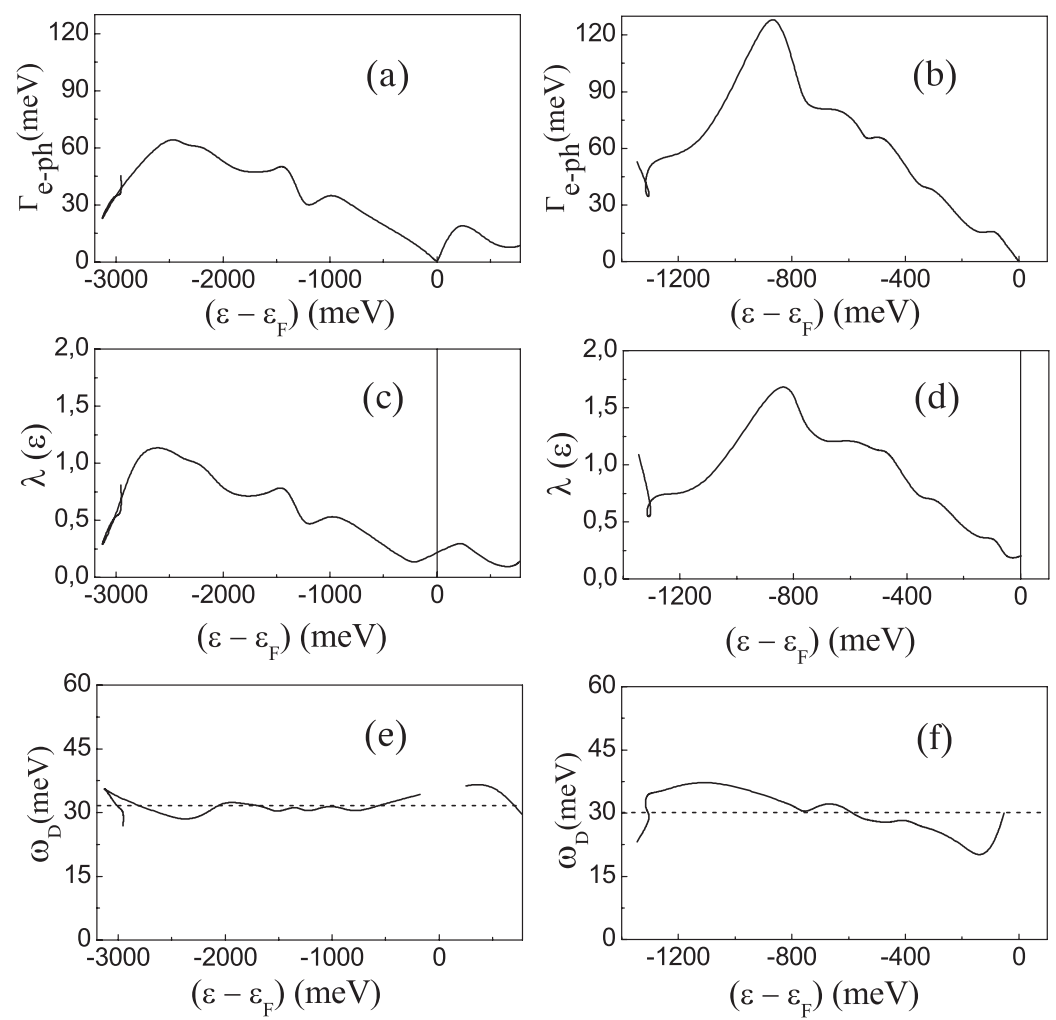

Figure 5. The $T=0$ phonon-induced lifetime broadening $\Gamma_{\mathrm{e}-\mathrm{ph}}$ and the electron-phonon coupling parameter $\lambda\left(\epsilon_{k_{i}}\right)$ as functions of energy for the $\Lambda_{1}((\mathrm{a}),(\mathrm{c}))$ and $\Lambda_{3}((\mathrm{~b}),(\mathrm{d}))$ energy bands, as well as the characteristic Debye frequency $\omega_{\mathrm{D}}((\mathrm{e}),(\mathrm{f}))$ calculated from equation $\Gamma_{\mathrm{e}-\mathrm{ph}}=2 \pi \lambda \hbar \omega_{\mathrm{D}} / 3$.

same direction. The last peak around $12-13 \mathrm{meV}$ is determined by the electron interaction with transverse modes from the $\Lambda$ symmetry direction. The values of $\lambda\left(\epsilon_{\boldsymbol{k}_{i}}\right)$ for electron states $W_{1}$ and $W_{1}^{\prime}$ do not differ substantially. The difference between their spectral functions is mainly determined by the high-energy contributions. For the state below the Fermi energy, $W_{1}$, the largest contributions come from the scattering of the transverse $\left(T_{2}\right)$ and longitudinal modes propagating along the $\Sigma$ direction while, in the case of the unoccupied $W_{1}^{\prime}$ state, the highenergy peak is determined by the e-ph coupling with the same phonon modes in the $Z$ direction and near the $X$ symmetry point.

For the states in the $\Lambda$ direction, the phonon-induced linewidth $\Gamma_{\mathrm{e}-\mathrm{ph}}$ changes more smoothly, especially in the case of the $\Lambda_{1}$ energy band (figure 5(a)), where there are no pronounced maxima and above the Fermi level $\Gamma_{\mathrm{e}-\mathrm{ph}}$ becomes more or less saturated. For the $\Lambda_{3}$ energy band, it first increases by a factor of four on moving to the centre of the symmetry direction and then goes slowly down to zero. In addition to the energy variation, the data also show a $\boldsymbol{k}$-dependence of the electron-phonon coupling, because we have different values of $\Gamma_{\mathrm{e}-\mathrm{ph}}\left(\epsilon_{k_{i}}\right)$ and $\lambda\left(\epsilon_{\boldsymbol{k}_{i}}\right)$ for electron states with the same energy and different momenta. On the whole, the obtained results show that the electron-phonon contribution to the total linewidth as well as the electron-phonon coupling parameter $\lambda\left(\epsilon_{k_{i}}\right)$ indeed depend strongly on both the $k$-position and the energy of an excited electron (hole) state.

The calculated dependence of $\Gamma_{\mathrm{e}-\mathrm{ph}}$ and $\lambda$ on the electron (hole) energy and momentum can be used for the determination of the characteristic Debye frequency, $\omega_{\mathrm{D}}$. It is well 

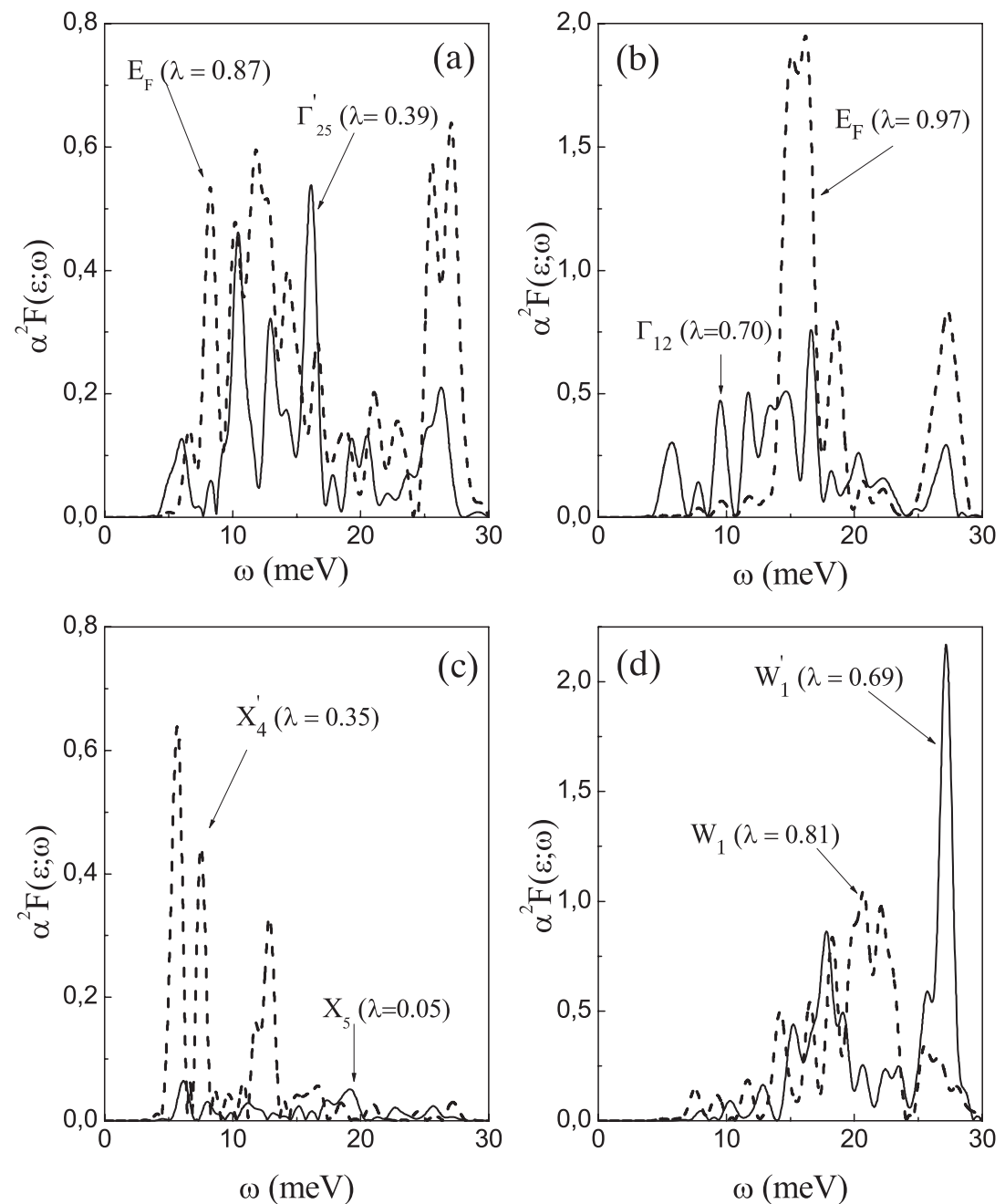

Figure 6. Electron-phonon spectral functions $\alpha^{2} F\left(\epsilon_{k_{i}} ; \omega\right)$ for some selected excited electron (hole) states along the $\Delta_{5}\left(\Gamma_{25}^{\prime}\right.$ and at $\left.\epsilon_{\mathrm{F}}\right)\left(\right.$ a) and $\Delta_{1}\left(\Gamma_{12}\right.$ and at $\left.\epsilon_{\mathrm{F}}\right)(\mathrm{b})$ energy bands, as well as at the $\mathrm{X}$ and $\mathrm{W}$ symmetry points.

known that the use of the Debye model for the phonon-induced contribution to the electron (hole) linewidth of surface states on metals leads to good agreement with experimental measurements [12-15, 17-20]. As was shown in [43], this model also works reasonably well in bulk metals. The Debye model relates $\Gamma_{\mathrm{e}-\mathrm{ph}}$ to $\lambda$ and $\omega_{\mathrm{D}}$ via an equation

$$
\Gamma_{\mathrm{e}-\mathrm{ph}}=2 \pi \lambda \hbar \omega_{\mathrm{D}} / 3
$$

derived at $T=0 \mathrm{~K}$ for electron (hole) energies $\left|\epsilon-\epsilon_{\mathrm{F}}\right|>\hbar \omega_{\mathrm{D}}[2,50]$. In the bottom panels of figures 4 and 5 we show the Debye frequency obtained from equation (8) with $\Gamma_{\mathrm{e}-\mathrm{ph}}$ and $\lambda$ shown in the upper panels of the same figures. We do not show $\omega_{\mathrm{D}}$ for $\left|\epsilon-\epsilon_{\mathrm{F}}\right|<\hbar \omega_{\mathrm{D}}$, since for these energies $\Gamma_{\mathrm{e}-\mathrm{ph}}$ depends on $\epsilon$, which makes the analysis more complicated [2, 50]. One can see that $\omega_{\mathrm{D}}$ varies noticeably with energy and momentum. The averaged $\omega_{\mathrm{D}}$ (dashed lines) for the bands in the $\Delta$ symmetry direction is equal to $\sim 32 \mathrm{meV}$. For the $\Lambda_{1}$ and $\Lambda_{3}$ energy 


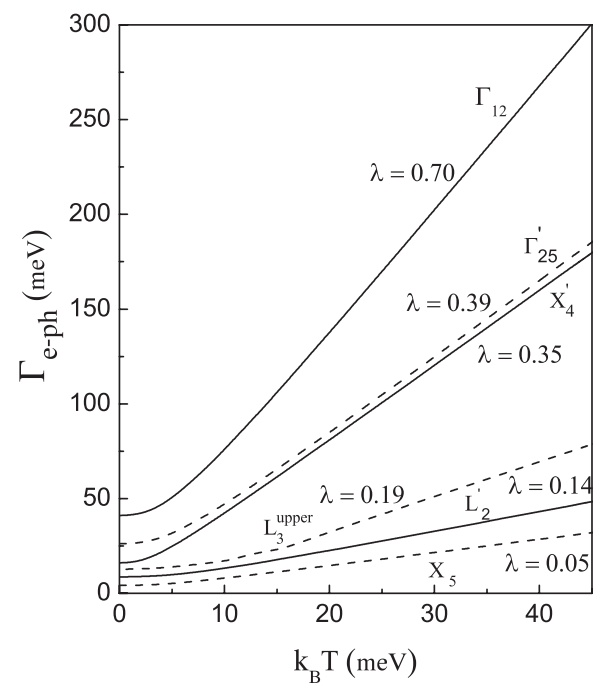

Figure 7. Temperature dependence of the phonon-induced lifetime broadening for the $\Gamma_{25}^{\prime}, \mathrm{L}_{3}, \mathrm{X}_{5}$ (dashed lines) and $\Gamma_{12}, \mathrm{~L}_{2}^{\prime}, \mathrm{X}_{4}^{\prime}$ (solid lines) states.

bands it is found to be $31 \mathrm{meV}$ and $30 \mathrm{meV}$, respectively. The calculated averaged value of $31 \mathrm{meV}$ is about $30 \%$ larger than the Debye frequency, $\omega_{\mathrm{D}}=23.7 \mathrm{meV}$, derived from specific heat measurements [51].

In figure 7 we show the temperature dependence of $\Gamma_{\mathrm{e}-\mathrm{ph}}$ for some electron (hole) states at the $\Gamma, \mathrm{L}$ and $\mathrm{X}$ symmetry points. For temperatures $k_{\mathrm{B}} T$ higher than the maximum phonon energy, the dependence becomes linear, with a slope that is determined by the electronphonon coupling parameter $\lambda\left(\epsilon_{k_{i}}\right)$. For the $\Gamma_{25}^{\prime}$ and $\mathrm{X}_{4}^{\prime}$ states, the curves are very close due to the almost equal values of $\lambda$. For the $\Gamma_{12}$ state, the steeper slope of the curve reflects the larger e-ph coupling. As is known, the inelastic electron (hole) linewidth $\Gamma_{\text {total }}$ can be represented as a sum, $\Gamma_{\text {total }}=\Gamma_{\mathrm{e}-\mathrm{ph}}+\Gamma_{\mathrm{e}-\mathrm{e}}$, where $\Gamma_{\mathrm{e}-\mathrm{e}}$ is an inelastic electron-electron contribution [5]. Usually, for energies $\left|\epsilon-\epsilon_{\mathrm{F}}\right| \geqslant 0.1 \mathrm{eV}, \Gamma_{\mathrm{e}-\mathrm{e}}$ is larger than $\Gamma_{\mathrm{e}-\mathrm{ph}}$, while for smaller energies, $\left|\epsilon-\epsilon_{\mathrm{F}}\right|<0.1 \mathrm{eV}, \Gamma_{\mathrm{e}-\mathrm{ph}}$ provides the dominant contribution to $\Gamma_{\text {total }}$. However, $\Gamma_{\mathrm{e}-\mathrm{ph}}$ varies strongly from one metallic system to another and, therefore, can play an important role for metals in which $\lambda$ is large. As follows from figures 4 and $5, \lambda$ in $\mathrm{Pd}$ is equal to 1.2-1.6 for some hole states in the $\Delta_{5}, \Delta_{1}$, and $\Lambda_{3}$ bands. These $\lambda$ s provide $\Gamma_{\mathrm{e}-\mathrm{ph}}$ which can be comparable with $\Gamma_{\mathrm{e}-\mathrm{e}}$ even at $T=0$. The $\Gamma_{\mathrm{e}-\mathrm{e}}$ averaged over momenta at a hole energy of $-1 \mathrm{eV}$ is $\sim 130 \mathrm{meV}$ (see ([52], [53])), while $\Gamma_{\mathrm{e}-\mathrm{ph}}$ at this energy and $T=0$ is $\sim 60 \mathrm{meV}$ for the $\Delta_{5}$ band, $\sim 90 \mathrm{meV}$ for the $\Delta_{1}$ band, and $\sim 100 \mathrm{meV}$ for the $\Lambda_{3}$ band. In contrast to $\Gamma_{\mathrm{e}-\mathrm{e}}$, which experiences very weak temperature dependence, the electronphonon contribution significantly increases with temperature (see figure 7). For instance, for the $\Lambda_{3}$ hole state at $\epsilon-\epsilon_{\mathrm{F}}=-1 \mathrm{eV}$, the electron-phonon contribution at room temperature is larger than $300 \mathrm{meV}$. Although $\Gamma_{\mathrm{e}-\mathrm{e}}$ varies with momentum, the comparison with the averaged $\Gamma_{\mathrm{e}-\mathrm{e}}$ clearly demonstrates the importance of the electron-phonon contribution to a hole linewidth in Pd even for the hole energies $\epsilon<\epsilon_{\mathrm{F}}-0.1 \mathrm{eV}$. As for the excited electrons, the comparison with available first-principles calculation results [52-55] for $\Gamma_{\mathrm{e}-\mathrm{e}}$ shows that, in general, $\Gamma_{\mathrm{e}-\mathrm{ph}}$ is significantly smaller than $\Gamma_{\mathrm{e}-\mathrm{e}}$ for energies $\epsilon>\epsilon_{\mathrm{F}}+0.1 \mathrm{eV}$ at $T=0$, while at room temperature $\Gamma_{\mathrm{e}-\mathrm{ph}}$ becomes comparable with $\Gamma_{\mathrm{e}-\mathrm{e}}$ for some excited states. 


\section{Summary and conclusions}

A first-principles study of the electron-phonon contribution to the phonon linewidths and to the lifetime broadening of electron and hole states in bulk Pd has been presented. An anomalously large broadening with strong $\boldsymbol{q}$ dependence was found for two phonon modes: the longitudinal branch along the $\Delta$ symmetry direction and $T_{1}$ transverse mode in the $\Sigma$ direction. These results agree well with the available experimental data [32,31]. In addition, the $T_{1}$ transverse modes are found to contribute most strongly to the electron-phonon scattering processes on the Fermi surface. We have also evaluated the electron-phonon coupling and its contribution to the lifetime broadening, $\Gamma_{\mathrm{e}-\mathrm{ph}}$, of the electron and hole states as functions of energy, momentum and temperature. The obtained results show strong dependence on both the $k$ position and the energy of an electron (hole) state. They also demonstrate the importance of the electronphonon contribution to the hole linewidth in Pd compared to the electron-electron contribution, $\Gamma_{\mathrm{e}-\mathrm{e}}$, even for large hole energies. As for the excited electrons, only at room temperature does $\Gamma_{\mathrm{e}-\mathrm{ph}}$ become comparable with $\Gamma_{\mathrm{e}-\mathrm{e}}$ for some excited states. In this connection, it is noteworthy that, in the study of the excited electron dynamics in the $\bar{\Gamma}$ surface state on $\operatorname{Pd}(111)$, Schäfer et al [56] found the discrepancy between the time-resolved two-photon-photoemission inverse lifetime measured at $\sim 400 \mathrm{~K}$ and the calculated $\Gamma_{\mathrm{e}-\mathrm{e}}$. This discrepancy, being $\sim 20 \mathrm{meV}$ and comparable to $\Gamma_{\mathrm{e}-\mathrm{e}}=37 \mathrm{meV}$, can be attributed to the e-ph contribution, which had not been included in the evaluation of $\Gamma_{\text {total }}$.

\section{Acknowledgments}

This work was partially supported by the University of the Basque Country, Departamento de Educación del Gobierno Vasco, MCyT (Grant No. MAT 2001-0946), and the European Community 6th Network of Excellence NANOQUANTA (NMP4-CT-2004-500198). Computational assistance and resources were partly provided by the SGI/IZO-SGIker at the UPV/EHU (supported by the Spanish Ministry of Education and Science and the European Social Fund).

\section{References}

[1] Abrikosov A A, Gorkov L R and Dzyaloshinski I E 1963 Methods of Quantum Field Theory in Statistical Physics (New York: Prentice-Hall/Englewood Cliffs)

[2] Grimvall G 1981 The electron-phonon interaction in metals Selected Topics in Solid State Physics ed E Wohlfarth (New York: North-Holland)

[3] Maksimov E G, Savrasov D Yu and Savrasov S Yu 1997 Sov. Phys._Usp. 40337

[4] Hellsing B, Eiguren A and Chulkov E V 2002 J. Phys.: Condens. Matter 145959

[5] Echenique P M, Berndt R, Chulkov E V, Fauster Th, Goldmann A and Höfer U 2004 Surf. Sci. Rep. 52219

[6] Eliashberg G M 1962 Sov. Phys._JETP 16780

[7] Wolf E L 1985 Principles of Electron Tunneling Spectroscopy (New York: Oxford University Press)

[8] Hengsberger M, Purdie D, Segovia P, Garnier M and Baer Y 1999 Phys. Rev. Lett. 83592

[9] Reinert F, Eltner B, Nicolay G, Ehm D, Schmidt S and Hüfner S 2003 Phys. Rev. Lett. 91186406

[10] LaShell S, Jensen E and Balasubramanian T 2000 Phys. Rev. B 612371

[11] Shi J, Tang S J, Wu B, Sprunger P T, Yang W L, Brouet V, Zhou X J, Hussain Z, Shen Z X, Zhang Z and Plummer E W 2004 Phys. Rev. Lett. 92186401

[12] McDougall B A, Balasubramanian T and Jensen E 1995 Phys. Rev. B 5113891

[13] Balasubramanian T, Jensen E, Wu X L and Hulbert S L 1998 Phys. Rev. B 57 R6866

[14] Hofmann Ph, Cai Y Q, Grütter Ch and Bilgram G H 1998 Phys. Rev. Lett. 811670

[15] Tang S-J, Ismail, Sprunger P T and Plummer E W 2002 Phys. Rev. B 65235428

[16] Eiguren A, Hellsing B, Reinert F, Nicolay G, Chulkov E V, Silkin V M, Hüfner S and Echenique P M 2002 Phys. Rev. Lett. 88066805 
[17] Gayone J E, Hofmann S V, Li Z and Hofmann Ph 2003 Phys. Rev. Lett. 91127601

[18] Paggel J J, Luh D-A, Miller T and Chiang T-C 2004 Phys. Rev. Lett. 92186803

[19] Kim T K, Sorensen T S, Wolfring E, Li H, Chulkov E V and Hofmann Ph 2005 Phys. Rev. B 72075422

[20] Kirkergaard C, Kim T K and Hofmann Ph 2005 New J. Phys. 799

[21] Smith N V, Thiry P and Petroff Y 1993 Phys. Rev. B 4715476

[22] Allen P B 1980 Dynamical Properties of Solids ed G K Norton and A A Maradudin (Amsterdam: North-Holland)

[23] McMillan W L 1968 Phys. Rev. 167331

[24] Allen P B 1972 Phys. Rev. B 62577

[25] Foner S, Doclo R and McNiff E J 1968 J. Appl. Phys. 39551

[26] Andersen O K 1970 Phys. Rev. B 2884

[27] Mueller F M, Freeman A J, Dimmock J O and Furdyna A M 1970 Phys. Rev. B 14617

[28] Butler W H 1977 Phys. Rev. B 155267

[29] Doniach S and Engelsberg S 1966 Phys. Rev. Lett. 17750

[30] Berk N F and Schrieffer J R 1966 Phys. Rev. Lett. 17433

[31] Miller A P 1975 Can. J. Phys. 532491

[32] Youngblood R, Noda Y and Shirane G 1979 Phys. Rev. B 196016

[33] Pinski F J and Butler W H 1979 Phys. Rev. B 196010

[34] Miller A P and Brockhouse B N 1968 Phys. Rev. Lett. 20798

[35] Baroni S, de Gironcoli S, Dal Corso A and Giannozzi P 2001 Rev. Mod. Phys. 73515

[36] Baroni S, de Gironcoli S, Dal Corso A and Giannozzi P http://www.pwscf.org

[37] Troullier N and Martins J L 1991 Phys. Rev. B 431993

[38] Perdew J and Zunger A 1981 Phys. Rev. B 235048

[39] Monkhorst H J and Pack J D 1976 Phys. Rev. B 135188

[40] Methfessel M and Paxton A T 1989 Phys. Rev. B 403631

[41] Savrasov S Y and Savrasov D Y 1996 Phys. Rev. B 5416487

[42] Dutton D H, Brockhouse B N and Miller A P 1972 Can. J. Phys. 502915

[43] Sklyadneva I Yu, Chulkov E V, Schöne W-D, Silkin V M, Keyling R and Echenique P M 2005 Phys. Rev. B 71174302

[44] Bauer R, Schmid A, Pavone P and Strauch D 1998 Phys. Rev. B 5711276

[45] Pinski F J, Allen P B and Butler W H 1978 Phys. Rev. Lett. 41431

[46] Himpsel F J and Eastman D E 1978 Phys. Rev. B 185236

[47] Andersen O K 1970 Phys. Rev. B 2883

[48] Christensen N E 1976 Phys. Rev. B 143446

[49] Papaconstantopoulos D A 1986 Handbook of Band Structure of Elemental Solids (New York: Plenum)

[50] Chulkov E V, Kliewer J, Berndt R, Silkin V M, Hellsing B, Crampin S and Echenique P M 2003 Phys. Rev. B $\mathbf{6 8} 195422$

[51] Ashcroft N W and Mermin N D 1976 Solid State Physics (Philadelphia, PA: Saunders College)

[52] Zhukov V P, Aryasetiawan F, Chulkov E V and Echenique P M 2002 Phys. Rev. B 65115116

[53] Zhukov V P, Chulkov E V and Echenique P M 2005 Phys. Rev. B 72155109

[54] Ladstädter F, de Pablos P F, Hohenester U, Pusching P, Ambrosch-Draxl C, de Andres P L, García-Vidal F and Flores F 2003 Phys. Rev. B 68085107

[55] Ladstädter F, Hohenester U, Pusching P and Ambrosch-Draxl C 2004 Phys. Rev. B 70235125

[56] Schäfer A, Shumay I L, Wiets M, Weinelt M, Fauster Th, Chulkov E V, Silkin V M and Echenique P M 2000 Phys. Rev. B 6113159 